\title{
三基色镍基自敏复合涂层的制备及其性能研究
}

\author{
何玲，李乾坤，李文生，崔帅 \\ (兰州理工大学 材料科学与工程学院, 省部共建有色金属先进加工与再利用国家重点实验室, 兰州 730050)
}

摘 要: 检测耐腐蚀涂层在腐蚀环境下的完整性, 对预测涂层工作寿命至关重要。通过电化学沉积法成功地将三基 色无机苂光颗粒: $\mathrm{BaMgAl}_{11} \mathrm{O}_{17}: \mathrm{Eu}^{2+}$ (蓝粉), $\mathrm{Y}_{2} \mathrm{O}_{3}: \mathrm{Eu}^{3+}$ (红粉), $\mathrm{CeMgAl}_{11} \mathrm{O}_{19}: \mathrm{Tb}^{3+}$ (绿粉)与金属镍复合, 在瓦特光亮 镀镍浴中沉积出具有三基色指示功能的镍基自敏复合涂层。采用 $\mathrm{X}$ 射线衍射(XRD)、扫描电镜(SEM)、表面轮廓仪、 维氏硬度计、荧光发射光谱和电化学测试手段分别对涂层组织形貌结构、润湿性、沉积率、硬度、发光性能及自 敏性能进行了检测，结果表明，阳离子表面活性剂十六烷基三甲基溴化铵(CTAB)和非离子表面活性剂聚乙二醇 (PEG)可以有效提高三基色荧光粒子沉积率。三种荧光粒子与金属镍之间表现出不同的润湿性和匹配性，使得其在 镍基复合涂层中具有不同的作用: $\mathrm{Y}_{2} \mathrm{O}_{3}: \mathrm{Eu}^{3+}$ 苂光粒子改变了涂层结晶取向, 且使晶粒细化, $\mathrm{BaMgAl}_{11} \mathrm{O}_{17}: \mathrm{Eu}^{2+}$ 使涂 层组织呈现节瘤状, $\mathrm{Y}_{2} \mathrm{O}_{3}: \mathrm{Eu}^{3+}$ 和 $\mathrm{CeMgAl}_{11} \mathrm{O}_{19}: \mathrm{Tb}^{3+}$ 粒子明显提高了涂层硬度。三基色荧光粒子在腐蚀前后表现出 不同的发光特性，可以有效指示出涂层工作周期内的完整性，起到良好的自敏检测作用。

关 键 词: 荧光颗粒; 电化学沉积; 自敏检测; 镍基复合涂层

中图分类号: TQ153 文献标识码: A

\section{Synthesis and Properties of Self-sensitization Luminescent Composite Coatings}

\author{
HE Ling, LI Qian-Kun, LI Weng-Sheng, CUI Shuai
}

(Lanzhou University of Technology, State Key Laboratory of Advanced Processing and Recycling of Non-ferrous Metals, School of Materials \& Science, Lanzhou 730050, China)

\begin{abstract}
It is very important for coating to detect integrity and working life in corrosive environment. The self-sensitization luminescent coating were fabricated by electroplating technique from a modified Watts bath combined with blue-emitting $\left(\mathrm{BaMgAl}_{11} \mathrm{O}_{17}: \mathrm{Eu}^{2+}\right)$, red-emitting $\left(\mathrm{Y}_{2} \mathrm{O}_{3}: \mathrm{Eu}^{3+}\right)$, and green-emtting $\left(\mathrm{CeMgAl}_{11} \mathrm{O}_{19}: \mathrm{Tb}^{3+}\right)$ rare-earth fluorescent particles. The surface morphology, microstructure, wettability, deposition rate, hardness, and self-sensitization luminescent property of the obtained composite coatings were characterized by using fluorescence spectrophotometer, surface profile measuring instrument, vickers, scanning electronmicroscope (SEM), X-ray diffraction (XRD), and electrochemical test techniques. The results show that surfactants of non-ionic PEG (polyethylene glycol) and cationic CTAB (cetyltrimethylammonium bromide) effectively improve the particles deposition rate. The fluorescent particles shows different wettability and matching with nickel, which have different effects on microstructure of the composite coatings. $\mathrm{Y}_{2} \mathrm{O}_{3}: \mathrm{Eu}^{3+}$ fluorescent particles change the coating crystal orientation and
\end{abstract}

收稿日期: 2016-04-12; 收到修改稿日期：2016-06-20

基金项目：国际科技合作专项项目(2015DFR51090); 甘肃省自然科学基金(1508RJZA049); 国家自然科学基金(51674130); 省部共建有色金属先进加工与再利用国家重点实验室开放基金(SKLAB02014010)

Key Program for International S\&T Cooperation Projects of China (2015DFR51090); Natural Science Foundation of Gansu Province (1508RJZA049); National Natural Science Foundation of China (51674130); Open fund of State Key Laboratory of Advanced Processing and Recycling of Non-ferrous Metals(SKLAB02014010)

作者简介：何 玲(1976-)，女，博士，教授. E-mail: hlswm@163.com 
grain refinement, $\mathrm{BaMgAl}_{11} \mathrm{O}_{17}: \mathrm{Eu}^{2+}$ fluorescent particles make the coating morphology presents knot. The coating hardness is increased obviously in composite coatings containing $\mathrm{Y}_{2} \mathrm{O}_{3}$ : $\mathrm{Eu}^{3+}$ and $\mathrm{CeMgAl}_{11} \mathrm{O}_{19}$ : $\mathrm{Tb}^{3+}$ particles. The fluorescent particles show different luminescent characteristics after corrosion, indicating good self-sensitization and effective monitor on the integrity of the composite coatings.

Key words: fluorescent particles; electrodeposition; self-sensitization monitor; Ni-base composite coatings

镍基涂层能够减少机械部件表面的腐蚀，提高 工件寿命, 得到了广泛应用 ${ }^{[1-4]}$ 。工件长期在腐蚀环 境工作下, 涂层不可避免会出现减薄、脱落, 造成涂 层和基体的破坏。因此, 对于腐蚀环境下工作的涂 层, 建立一种早期预警指示机制来检测涂层在工作 周期内的完整性至关重要。研究显示, 将苂光颗粒 与镍共沉积, 能够有效提高涂层的硬度和耐腐蚀性 能，并实现预警指示效果。Feldstein 等 ${ }^{[5]}$ 首次实现了 镍与苂光颗粒的化学沉积; Eldridge 等 ${ }^{[6]}$ 利用电子束 物理气相沉积将荧光颗粒沉积在航空发动机热障涂 层表面，实现了荧光颗粒与金属涂层的分层性指示; Ganapathi 等 ${ }^{[7]}$ 在有机融熔液体(乙酰胺和二甲基钒) 浴中电沉积金属与菼光颗粒。上述制备工艺中镀液 的准备过程繁琐, 设备昂贵, 荧光粒子在沉积过程 中会出现热猝灭, 降低苂光粒子的发光特性, 影响 涂层的自敏指示特性。针对以上问题, $\mathrm{He}$ 等 ${ }^{[8]}$ 在较 低温度下利用电化学沉积法在碳钢表面制备了蓝光 指示性涂层, 并对其发光性能进行了研究。但是单 一色彩自敏涂层难以满足不同腐蚀工况条件下的指 示需求。稀土发光材料亮度高、发光稳定、寿命长, 已广泛应用于照明、生物等领域 ${ }^{[9-12]}$ 。本工作采用 电化学沉积法, 将商用三基色荧光颗粒 $\left(\mathrm{BaMgAl}_{11} \mathrm{O}_{17}: \mathrm{Eu}^{2+} 、 \mathrm{Y}_{2} \mathrm{O}_{3}: \mathrm{Eu}^{3+} 、 \mathrm{CeMgAl}_{11} \mathrm{O}_{19}: \mathrm{Tb}^{3+}\right)$ 与镍复合沉积在不锈钢表面, 形成三基色发光自敏 复合涂层, 并对三种荧光粒子与金属镍之间的润湿 匹配性、复合涂层的组织结构, 以及在腐蚀环境中 涂层发光衰减特性的差异进行了研究。

\section{1 实验方法}

\section{1 试剂与仪器}

试验以六水合硫酸镍、氯化镍、嶰酸、1-4 丁 炔二醇、香豆素、十六烷基三甲基溴化铵(CTAB)、

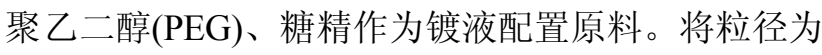
$5 \sim 10 \mu \mathrm{m}$ 的稀土三基色苂光粉颗粒(蓝光- $\mathrm{BaMgAl}_{11} \mathrm{O}_{17}$ : $\mathrm{Eu}^{2+}$, 绿光 $-\mathrm{CeMgAL}_{11} \mathrm{O}_{19}: \mathrm{Tb}^{3+}$, 红光 $-\mathrm{Y}_{2} \mathrm{O}_{3}: \mathrm{Eu}^{3+}$ )与涂 层复合, 采用纯镍片作为阳极 $(90 \mathrm{~mm} \times 10 \mathrm{~mm})$, 以抛光后的 SUS304 不锈钢片 $(90 \mathrm{~mm} \times 10 \mathrm{~mm})$ 作为 阴极, 将磷酸三钠、氢氧化钠、无水碳酸钠、硅酸 钠、盐酸(质量分数为 $36 \%$ 38\%)作为基体预处理的 原料。

实验利用 DF-101 集热式恒温加热磁力摚拌器 进行搅拌, CHI660D 电化学工作站进行电沉积和腐 蚀测试。在 F97 苂光分光光度计对涂层发光性能进 行检测, 在维氏硬度计上测量涂层硬度, 利用 D8/ADVANCE 型 X 射线衍射仪和 JEOL-6700F 型 扫描电子显微镜对试样的物相、微观结构和表面形 貌进行分析。使用荧光显微镜中多相组织分析手段, 统计发光荧光粒子在涂层中所占的体积分数, 测算 粒子在复合涂层当中的沉积率。

\section{2 实验过程}

(1) 基体预处理

将打磨抛光好的不锈钢试片置于磷酸三钠、氢 氧化钠、无水碳酸钠与硅酸钠配置成的有机除油溶 液中, 在 $60^{\circ} \mathrm{C}$ 下超声波清洗, 再用蒸馏水冲洗后放 入干燥箱以待用。

(2) 镀液配置

将六水合硫酸镍 $\left(\mathrm{NiSO}_{4} \cdot 6 \mathrm{H}_{2} \mathrm{O}\right)$ 、六水合氯化镍 $\left(\mathrm{NiCl}_{2} \cdot 6 \mathrm{H}_{2} \mathrm{O}\right)$ 、嗍酸 $\left(\mathrm{H}_{3} \mathrm{BO}_{3}\right)$ 、糖精(Saccharin)、1-4 丁 炔二醇(1, 4-butynediolcoumarin)、香豆素(Coumarin), 阳 离子表面活性剂十六烷基三甲基溴化铵(CTAB)和非离 子表面活性剂聚乙二醇(PEG)一起加入去离子水中, 溶 解后作为镀液溶液, 然后添加稀土三基色苂光粉颗粒 (蓝光- $\mathrm{BaMg}-\mathrm{Al}_{11} \mathrm{O}_{17}: \mathrm{Eu}^{2+}$, 绿光 $-\mathrm{CeMgAL}_{11} \mathrm{O}_{19}: \mathrm{Tb}^{3+}$, 红光 $\left.-\mathrm{Y}_{2} \mathrm{O}_{3}: \mathrm{Eu}^{3+}\right)$, 并将镀液超声分散, 镀液成分如表 1 所示。

表 1 电解液成份

Table1 Chemical composition of the electrodeposition bath

\begin{tabular}{ccccccccc}
\hline Composition & $\mathrm{NiSO}_{4} \cdot 6 \mathrm{H}_{2} \mathrm{O}$ & $\mathrm{NiCl}_{2} \cdot 6 \mathrm{H}_{2} \mathrm{O}$ & $\mathrm{H}_{3} \mathrm{BO}_{3}$ & Saccharin & 1, 4-butynediolcoumarin & $\mathrm{Coumarin}$ & $\mathrm{CTAB}$ & $\mathrm{PEG}$ \\
\hline $\begin{array}{c}\text { Concentration/ } \\
\left(\mathrm{g} \cdot \mathrm{L}^{-1}\right)\end{array}$ & 250 & 45 & 40 & 1 & 0.3 & 0.1 & $0.1 / 0.2$ & 0.04 \\
\hline
\end{tabular}


(3) 电镀

荧光粉颗粒浓度为 $15 \sim 40 \mathrm{~g} / \mathrm{L}$, 采用 $\mathrm{NaOH}$ $(0.1 \mathrm{~mol} / \mathrm{L})$ 和 $\mathrm{H}_{2} \mathrm{SO}_{4}(0.1 \mathrm{~mol} / \mathrm{L})$ 将镀液 $\mathrm{pH}$ 调节至 4.0 , 将预处理好的不锈钢试片在 $\mathrm{HCl}$ 溶液浸泡 $60 \mathrm{~s}$ 以除去表面氧化层。镀液在 $50^{\circ} \mathrm{C}$ 水浴中磁力搅拌, 摚拌速率为 $400 \mathrm{r} / \mathrm{min}$, 电镀时间为 $40 \mathrm{~min}$, 电流密 度为 $70 \mathrm{~mA} / \mathrm{cm}^{2}$ 。

\section{2 结果与讨论}

\section{1 三基色荧光粒子的沉积率及其润湿性}

三基色苂光颗粒的种类及其在镀液中的浓度对 粒子在复合涂层中的沉积率都有较大影响。如图 1 所示, 由于三种荧光粉比重不同, 当荧光颗粒浓度 升高时表现出不同的沉积特性。 $\mathrm{BaMgAl}_{11} \mathrm{O}_{17}: \mathrm{Eu}^{2+}$ 和 $\mathrm{Y}_{2} \mathrm{O}_{3}: \mathrm{Eu}^{3+}$ 沉积率出现先升后降的特性, 这是由 于随着苂光颗粒浓度提高, 颗粒间团聚几率增大, 粒子会出现沉降而不能良好地悬浮在镀液中, 因此 浓度为 $20 \mathrm{~g} / \mathrm{L}$ 时, $\mathrm{BaMgAl}_{11} \mathrm{O}_{17}: \mathrm{Eu}^{2+}$ 和 $\mathrm{Y}_{2} \mathrm{O}_{3}: \mathrm{Eu}^{3+}$ 荧光颗粒的沉积率最大, 分别为 $64.1 \%$ 和 $31.6 \%$ 。而 $\mathrm{CeMgAl}_{11} \mathrm{O}_{19}$ : $\mathrm{Tb}^{3+}$ 比重大于前两者, 在相同浓度下 镀液中的颗粒数最少, 发生团聚的几率减小, 因此 随着浓度提高, 粒子的沉积率呈现上升趋势。三种 苂光颗粒在复合涂层中的沉积特性不同的另外一个 原因是由于阳离子表面活性剂(CTAB) 使苂光颗粒 表面形成带正电的离子氛, 而三种荧光颗粒 Zeta 电 位的不同, 使粒子在相同浓度下沉积特性有所差 异。综合考虑, 荧光颗粒浓度为 $20 \mathrm{~g} / \mathrm{L}$ 时, 复合涂 层中的粒子的沉积性能最佳。

图 2 给出了三基色苂光粒子沉积率与阳离子表 面活性剂 CTAB 浓度之间的关系。随着 CTAB 浓度

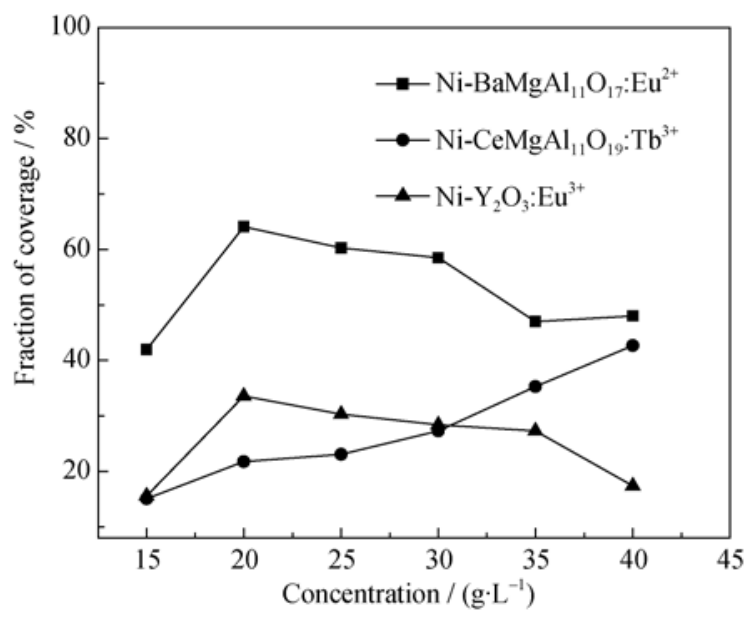

图 1 不同浓度镀液中三基色苂光颗粒在复合涂层的沉积率

Fig. 1 Deposition rate of fluorescence particles on the composite coatings in the plating bath with different concentrations

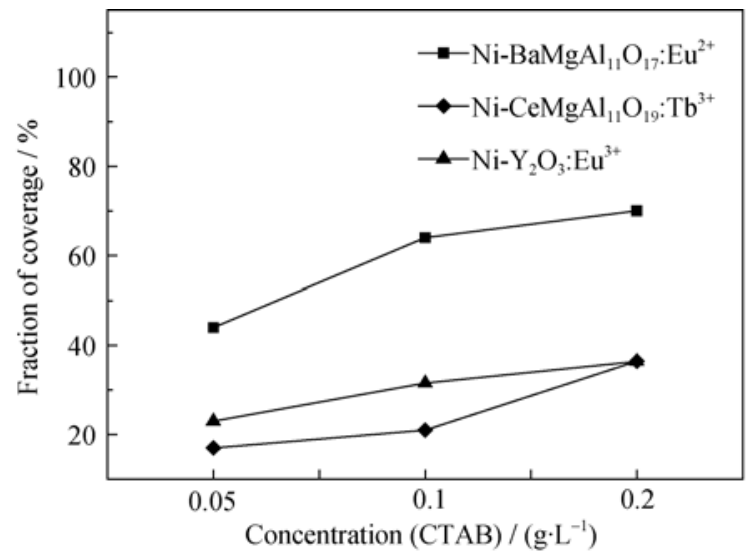

图 2 表面活性剂(CTAB)浓度对粒子沉积率的影响

Fig. 2 Effect of surfactant (CTAB) concentration on deposition rate of fluorescence particles

升高, 荧光粒子沉积率明显上升, 当 $\mathrm{CTAB}$ 浓度为 $0.2 \mathrm{~g} / \mathrm{L}$ 时, 三种荧光粒子沉积率都达到最大, 分别 为 $70 \%\left(\mathrm{BaMgAl}_{11} \mathrm{O}_{17}: \mathrm{Eu}^{2+}\right) 、 36.5 \%\left(\mathrm{CeMgAl}_{11} \mathrm{O}_{19}\right.$ : $\left.\mathrm{Tb}^{3+}\right) 、 36.4 \%\left(\mathrm{Y}_{2} \mathrm{O}_{3}: \mathrm{Eu}^{3+}\right)$, 继续增大 $\mathrm{CTAB}$ 的浓度 会出现涂层开裂现象。CTAB 浓度的升高一方面使 更多的苂光粒子表面富集正电荷，从而提高粒子在 镀液中的悬浮量; 另一方面当过多的苂光粒子停留 在电极表面时，粒子之间容易发生团聚，使涂层表 面荧光粒子的沉积出现不均匀现象。非离子表面活 性剂 PEG 在电沉积过程中对电极有极化作用，提供 沉积阻力, 加入少量 PEG 后, PEG 的疏水基团吸附 在荧光粒子表面，亲水基团朝向水分子，利用 $\mathrm{PEG}$ 长链起到空间位阻效应，减少苂光粒子之间的团聚， 最终使苂光粒子均匀沉积在电极表面。

图 3 给出了荧光粒子添加浓度为 $20 \mathrm{~g} / \mathrm{L}$, 表面 活性剂浓度为 $\operatorname{CTAB}(0.1 \mathrm{~g} / \mathrm{L})$ 时, 三种荧光粒子和镍 离子在沉积过程中的过电位曲线。三种荧光粒子对

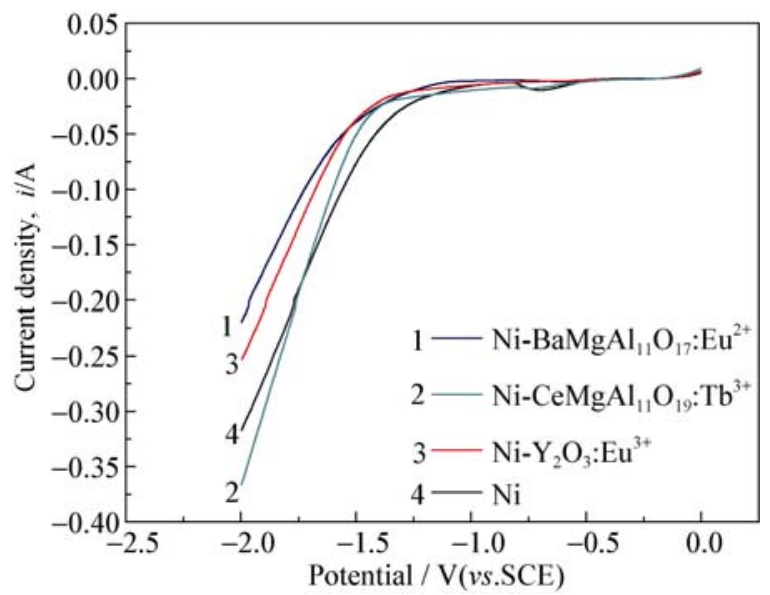

图 3 不同复合涂层线性伏安曲线

Fig. 3 Curves of linear sweep voltammetry of different composite coatings 
$\mathrm{CTAB}$ 的吸附能力不同导致菼光粒子在电极表面 过电位的差异，从而引起颗粒润湿性的不同。同时 荧光粒子的吸附与 $\mathrm{Ni}$ 的结晶生长存在竞争机制, 粒子吸附在电极表面, 同时 $\mathrm{Ni}^{2+}$ 在阴极的还原作用 下析出。如图 3 所示, $\mathrm{BaMgAl}_{11} \mathrm{O}_{17}: \mathrm{Eu}^{2+}$ 和 $\mathrm{Y}_{2} \mathrm{O}_{3}$ : $\mathrm{Eu}^{3+}$ 粒子的过电位与镍离子相比负移, 这是由于表 面吸附了 CTAB 后, 上述两种荧光粒子在电极表面 优先占据 $\mathrm{Ni}$ 结晶形核位置, 阻碍了镍离子的电子 转移, 使得荧光颗粒优先沉积, 表明在沉积过程中 蓝色和红色苂光粒子具有良好的润湿性。蓝色荧光 颗粒 $\mathrm{BaMgAl}_{11} \mathrm{O}_{17}: \mathrm{Eu}^{2+}$ 的过电位负移程度大于红 色荧光颗粒 $\mathrm{Y}_{2} \mathrm{O}_{3}$ : $\mathrm{Eu}^{3+}$ 。绿色荧光颗粒 $\mathrm{CeMgAl}_{11} \mathrm{O}_{19}: \mathrm{Tb}^{3+}$ 的过电位相比纯镍涂层有所正 移, 润湿效果不明显。

\section{2 三基色荧光粒子对涂层结构和硬度的影响}

三种荧光粒子浓度为 $20 \mathrm{~g} / \mathrm{L}$ 时, 与镍共沉积后 涂层的 SEM 表面形貌如图 4 所示。由图可知三基色 发光复合涂层表面较为致密, 荧光粒子与涂层结合 牢固, 未出现表面脱落的痕迹, 局部地方有少量氢 气在电极表面发生吸脱附而留下的凹坑。 $\mathrm{Ni}-\mathrm{BaMgAl}_{11} \mathrm{O}_{17}: \mathrm{Eu}^{2+}$ 复合涂层中荧光粒子沉积率 较高, 涂层表面荧光粒子出现局部团聚, $\mathrm{Ni}-\mathrm{CeMgAl} \mathrm{H}_{11} \mathrm{O}_{19}: \mathrm{Tb}^{3+}$ 和 $\mathrm{Ni}-\mathrm{Y}_{2} \mathrm{O}_{3}: \mathrm{Eu}^{3+}$ 涂层中荧光 粒子分布均匀, 分散性良好。

图 5 为三基色复合涂层的断面形貌, 图中三基 色发光复合涂层与基体结合牢固, 界面处没有出现 裂缝。在涂层纵向, 苂光颗粒的分布呈现差异性。
电极表面，随着沉积过程进行，较小的颗粒嵌入涂 沉积过程按照 Stokes 定律, 较大的颗粒优先沉积在 层表层。图 5(a)中发现 $\mathrm{BaMgAl}_{11} \mathrm{O}_{17}: \mathrm{Eu}^{2+}$ 颗粒的沉 积改变了涂层的结晶形貌, 出现节瘤状组织, 这是 由于沉积率较大时荧光粒子发生团聚, 阻碍了 $\mathrm{Ni}$ 结 晶形核和长大, 导致 $\mathrm{Ni}$ 结晶位置电流密度升高, 造 成局部 $\mathrm{Ni}$ 晶粒迅速长大出现节瘤状组织。从图 5(b,c) 可以看出, $\mathrm{CeMgAl}_{11} \mathrm{O}_{19}: \mathrm{Tb}^{3+}$ 和 $\mathrm{Y}_{2} \mathrm{O}_{3}: \mathrm{Eu}^{3+}$ 苂光粒子 均匀分布在涂层中, 涂层致密, 没有节瘤状组织, 说 明苂光粒子的吸附与 $\mathrm{Ni}$ 结晶生长相互竞争达到平衡。

发光复合涂层的 XRD 图谱如图 6 所示, 图中 (200)和(111)衍射峰对应于纯镍的衍射峰, 与镍相衍 射峰强度比较, 苂光粒子衍射强度较弱。XRD 图谱 中未发现其他杂相生成, 说明荧光粒子没有与 $\mathrm{Ni}$ 发 生反应。在涂层中分别添加 $\mathrm{BaMgAl}_{11} \mathrm{O}_{17}: \mathrm{Eu}^{2+}$ 和 $\mathrm{CeMgAl}_{11} \mathrm{O}_{19}: \mathrm{Tb}^{3+}$ 颗粒后, $\mathrm{Ni}$ 的晶体结构没有改变。 但是在涂层中添加 $\mathrm{Y}_{2} \mathrm{O}_{3}$ : $\mathrm{Eu}^{3+}$ 颗粒后, $\mathrm{Ni}$ 相的结晶 取向由(200)晶面向(111)发生转变, 这是由于电沉积 过程中 $\mathrm{Y}_{2} \mathrm{O}_{3}: \mathrm{Eu}^{3+}$ 颗粒改变了 $\mathrm{Ni}(\mathrm{OH})_{2}$ 和活性氢原 子的吸附作用，进而抑制了 $\mathrm{Ni}$ 相(200)生长方向 ${ }^{[13]}$ 。 根据 Scherrer 方程计算三种涂层的晶粒大小:

$$
D=\mathrm{K} \lambda / \mathrm{FW}(S) \times \cos (\theta)
$$

式中 $\lambda 、 \mathrm{FW}(S) 、 \theta$ 分别代表 $\mathrm{Cu}-\mathrm{K} \alpha$ 的波长、卷积大 小、衍射角, $D$ 为晶粒大小, $\mathrm{K}$ 在涂层中一般取 0.5 。 计算结果表明, 三种苂光粒子的晶粒大小分别为 $21.4 \mathrm{~nm}\left(\mathrm{BaMgAl}_{11} \mathrm{O}_{17}: \mathrm{Eu}^{2+}\right) 、 18 \mathrm{~nm}\left(\mathrm{CeMgAl}_{11} \mathrm{O}_{19}\right.$ : $\left.\mathrm{Tb}^{3+}\right) 、 10.4 \mathrm{~nm}\left(\mathrm{Y}_{2} \mathrm{O}_{3}: \mathrm{Eu}^{3+}\right)$ 。说明 $\mathrm{Y}_{2} \mathrm{O}_{3}: \mathrm{Eu}^{3+}$ 颗粒中
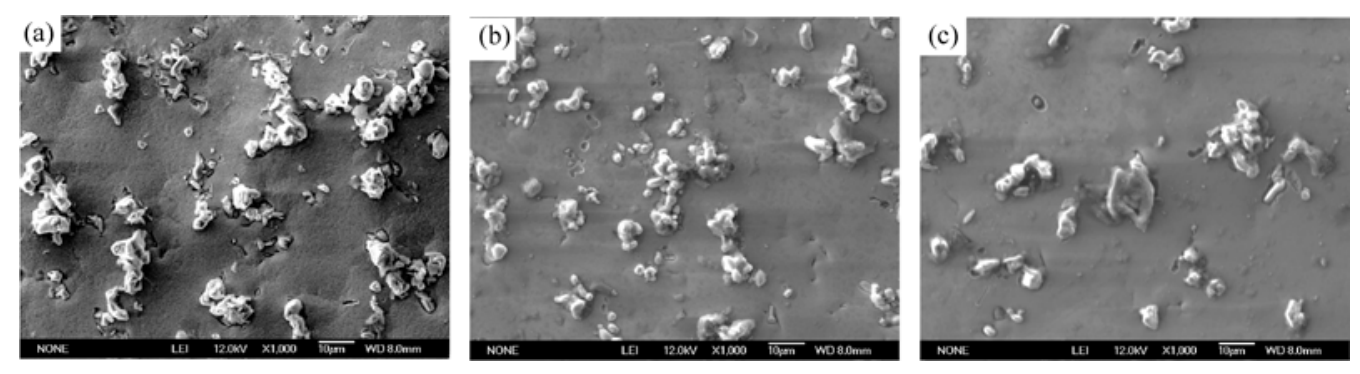

图 4 三基色发光复合涂层的表面 SEM 照片

Fig. 4 Surface morphologies of composite coatings (a) $\mathrm{Ni}-\mathrm{BaMgAl}_{11} \mathrm{O}_{17}: \mathrm{Eu}^{2+}$; (b) $\mathrm{Ni}-\mathrm{CeMgAl}_{11} \mathrm{O}_{19}: \mathrm{Tb}^{3+}$; (c) $\mathrm{Ni}^{-} \mathrm{Y}_{2} \mathrm{O}_{3}: \mathrm{Eu}^{3+}$
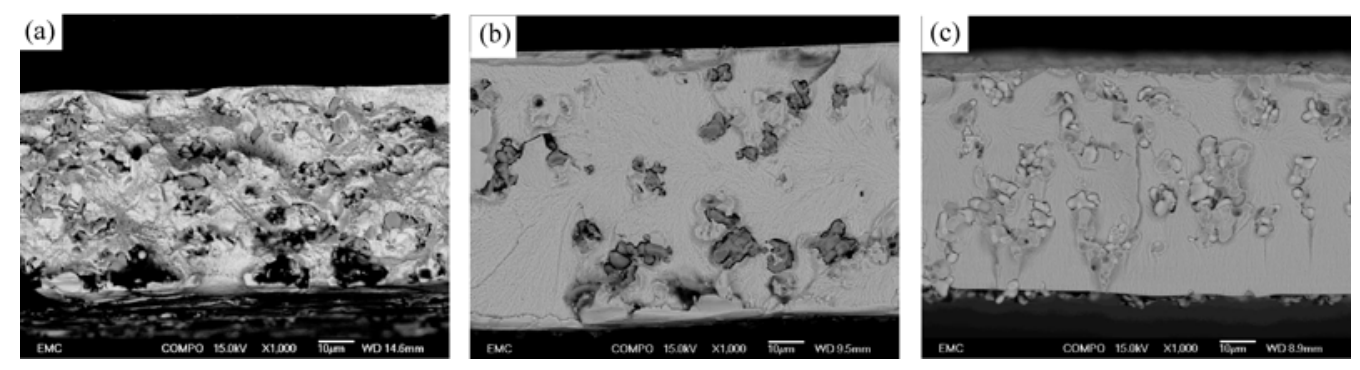

图 5 三基色发光复合涂层的断面形貌照片

Fig. 5 Cross-sectional optical micrographs of composite coatings (a) $\mathrm{Ni}-\mathrm{BaMgAl}_{11} \mathrm{O}_{17}: \mathrm{Eu}^{2+}$; (b) $\mathrm{Ni}-\mathrm{CeMgAl}_{11} \mathrm{O}_{19}: \mathrm{Tb}^{3+}$; (c) $\mathrm{Ni}-\mathrm{Y}_{2} \mathrm{O}_{3}: \mathrm{Eu}^{3+}$ 


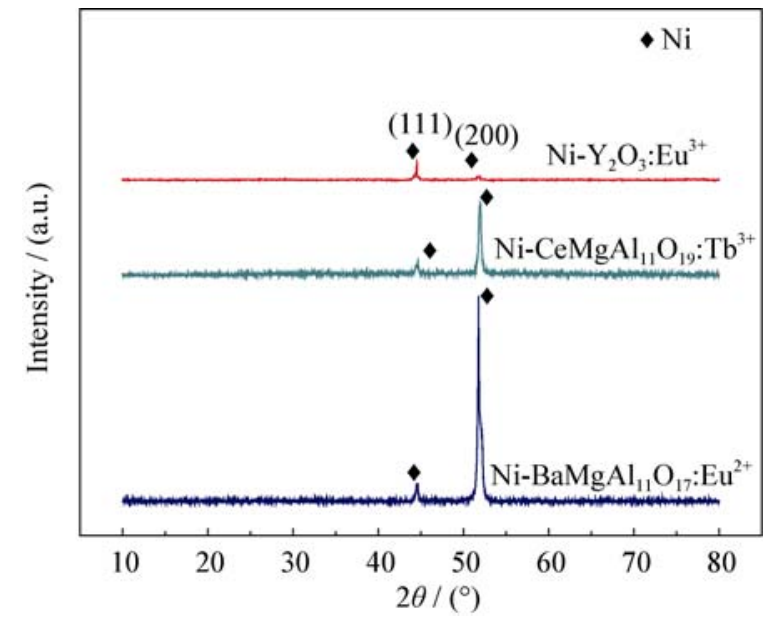

图 6 三基色发光复合涂层的 XRD 图谱

Fig. 6 XRD patterns of composite coatings

的钎原子具有钉扎镍结晶边界的作用 ${ }^{[14-17]}$, 阻碍了 镍原子扩散长大, 从而使 $\mathrm{Ni}-\mathrm{Y}_{2} \mathrm{O}_{3} \mathrm{Eu}^{3+}$ 涂层的 $\mathrm{Ni}$ 晶 粒最小。

复合涂层硬度与第二相粒子的属性密切相关, 图 7 给出了荧光粒子添加浓度对复合涂层硬度的 影响。由图 7 可知, 随粒子浓度升高, 涂层硬度增 大，增大趋势与粒子沉积率变化趋势一致。这表明 影响涂层硬度的大小与苂光粒子的沉积率有密切 关系, 苂光粒子沉积率越大其涂层硬度越高。荧光 粒子属于陶瓷无机颗粒, 其本身就具有很高的硬 度，陶瓷颗粒对涂层会起到弥散强化的作用而使

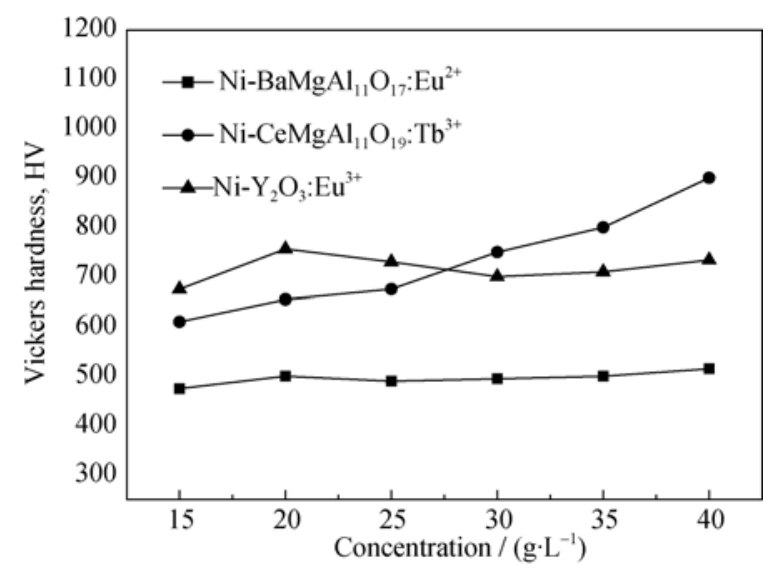

图 7 苂光粒子添加浓度对复合涂层硬度的影响

Fig. 7 Effect of concentration of fluorescence particles on the hardness of coatings
涂层硬度明显升高。

从系列样品中选取相近沉积率(35\% 42\%)的三 种复合涂层，给出了相近沉积率下复合涂层硬度、 粗䊅度等相关参数，如表 2 所示。由表可知，三种苂 光粒子对涂层表面一致性产生了不同的影响。红光 复合涂层硬度最高, 表面粗粘度 $R_{\mathrm{a}}$ 和 $R_{\max }$ 较大, 这 是由于 $\mathrm{Y}_{2} \mathrm{O}_{3}: \mathrm{Eu}^{3+}$ 粒子自身具有高硬度, 涂层晶粒 最小而起到细晶强化的作用, 并且 $\mathrm{Y}_{2} \mathrm{O}_{3}: \mathrm{Eu}^{3+}$ 颗粒 中的钇原子具有钉扎镍结晶边界的作用，可以有效 抑制位错源的移动, 导致红光涂层在三种涂层中的 硬度最高, 且红光涂层中 $\mathrm{Y}_{2} \mathrm{O}_{3}: \mathrm{Eu}^{3+}$ 颗粒中的钇原子 会抑制晶粒长大，使晶粒沿纵向生长趋势增大，表 面粗楉度增加。而绿光复合涂层 $\left(\mathrm{Ni}-\mathrm{CeMgAl}_{11} \mathrm{O}_{19}\right.$ : $\mathrm{Tb}^{3+}$ ) 硬度仅次于红光复合涂层, 且粗䊁度最小, 这 是因为 $\mathrm{CeMgAl}_{11} \mathrm{O}_{19}$ : $\mathrm{Tb}^{3+}$ 自身含有两种稀土元素 $\mathrm{Ce}$ 与 $\mathrm{Tb}$, 两种稀土元素充当惰性稀土相可以细化 晶粒，在涂层中也起到抑制位错源的作用 ${ }^{[18-19]}$, 使 得涂层在结晶中趋于平整，涂层粗䊁度较小，表面 轮廓起伏较小, 因此涂层硬度仅次于红光复合涂 层。蓝光复合涂层中 $\mathrm{BaMgAl}_{11} \mathrm{O}_{17}: \mathrm{Eu}^{2+}$ 颗粒自身硬 度较低, 粒子不具有细化晶粒的作用, 使得涂层晶 粒粗大, 平均粗粘度 $R_{\mathrm{a}}$ 较大，涂层表面粗粘，表面 起伏较大。

\section{3 三基色发光自敏复合涂层的发光性能}

图 8 为三基色发光复合涂层荧光粒子在 $254 \mathrm{~nm}$ 紫外光激发下的发射光谱。图 8(a)为 $\mathrm{Eu}^{2+}$ 在六方结 构 $\mathrm{BaMgAl}_{11} \mathrm{O}_{17}$ 基质晶格中的发光特征, 可见 $\mathrm{Eu}^{2+}$ 取代了镜面层 $\mathrm{Ba}^{2+}$ 的格位形成的发光中心，在晶体 场作用下发生 $4 \mathrm{f}^{6} 5 \mathrm{~d}^{1}-4 \mathrm{f}^{7}$ 跃迁, 形成 $452 \mathrm{~nm}$ 的蓝光 发射。图 8(b)为 $\mathrm{Tb}^{3+}$ 在 $\mathrm{CeMgAl}_{11} \mathrm{O}_{19}$ 基质中分别出 现在 490、542、590、620 nm 的 ${ }^{5} \mathrm{D}_{4} \rightarrow{ }^{7} \mathrm{~F}_{5}$ 发射。图 $8(\mathrm{c})$ 为 $\mathrm{Eu}^{3+}$ 在 $\mathrm{Y}_{2} \mathrm{O}_{3}$ 基质晶格中出现了主峰位于 $611 \mathrm{~nm} \mathrm{Eu}{ }^{3+}$ 的 ${ }^{5} \mathrm{D}_{0} \rightarrow{ }^{7} \mathrm{~F}_{2}$ 的发射。从图 8 可以看出, 涂 层中荧光粒子的发光强度低于其本征发光强度, 说 明苂光颗粒的发光在涂层中出现了猝灭现象。电化 学沉积法在低温下进行, 荧光粒子在沉积过程中不 受温度的影响, 荧光粒子不会发生热猝灭。因此涂 层中荧光粒子与金属 $\mathrm{Ni}$ 接触后, 有可能发生了接触 性猝灭效应，使苂光发射强度下降。

表 2 复合涂层相关参数

Table 2 Properties of the coatings studied

\begin{tabular}{ccccc}
\hline Coating & Grain size $/ \mathrm{nm}$ & Microhardness, HV & Roughness parameters, $R_{\mathrm{a}} / \mu \mathrm{m}$ & Roughness parameters, $R_{\max } / \mu \mathrm{m}$ \\
\hline $\mathrm{Ni}-\mathrm{BaMgAl}_{11} \mathrm{O}_{17}: \mathrm{Eu}^{2+}$ & 23.5 & 530 & 1.389 & 7.50 \\
$\mathrm{Ni}-\mathrm{CeMgAl}_{11} \mathrm{O}_{19}: \mathrm{Tb}^{3+}$ & 17.1 & 745 & 0.847 & 3.96 \\
$\mathrm{Ni}-\mathrm{Y}_{2} \mathrm{O}_{3}: \mathrm{Eu}^{3+}$ & 11.1 & 756 & 1.296 & 7.60 \\
\hline
\end{tabular}



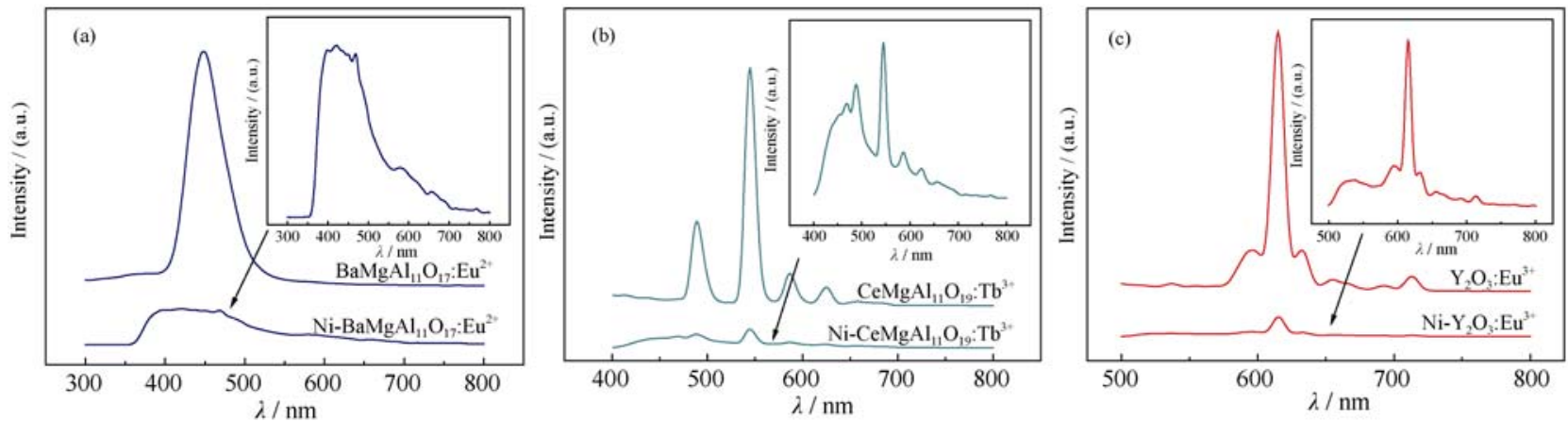

图 8 三基色发光复合涂层的发射光谱图

Fig. 8 Emission spectra of composite coatings (a) $\mathrm{Ni}-\mathrm{BaMgAl}_{11} \mathrm{O}_{17}: \mathrm{Eu}^{2+}$; (b) $\mathrm{Ni}-\mathrm{CeMgAl}_{11} \mathrm{O}_{19}: \mathrm{Tb}^{3+}$; (c) $\mathrm{Ni}-\mathrm{Y}_{2} \mathrm{O}_{3}: \mathrm{Eu}^{3+}$

图 9 给出了腐蚀前后三基色发光复合涂层的发 射光谱，由图可知，腐蚀后涂层中的荧光颗粒发射 峰位置没有改变, 但是发光强度减弱, 这是由于苂 光粒子作为非导电介质, 在腐蚀过程中是一种有效 抵抗腐蚀的屏蔽介质，起到阻挡腐蚀路径的作用。 蓝光和绿光复合涂层发光强度减弱程度较小, 红光 复合涂层发光强度减弱明显。在 $\mathrm{Ni}-\mathrm{Y}_{2} \mathrm{O}_{3}: \mathrm{Eu}^{3+}$ 复合 涂层中, $\mathrm{Ni}$ 相的结晶取向由(200)面转变为(111)面, (111)晶面作为密排面比(200)晶面具有更好的耐腐 蚀效果, 从而使 $\mathrm{Y}_{2} \mathrm{O}_{3}: \mathrm{Eu}^{3+}$ 受到较多的腐蚀而造成 发光减弱明显的现象。所以在长期腐蚀的工况条件 下，蓝光和绿光复合涂层在经过腐蚀后的发光衰减 特性较慢, 自敏指示效果长久, 可以用于评价涂层 长期腐蚀后的完整性。

\section{3 结论}

1) 采用低温电化学沉积法成功制备了三基色 镍基自敏发光指示涂层, 发现荧光粒子的种类和浓
度对粒子沉积率产生了不同的影响, $\mathrm{BaMgAl}_{11} \mathrm{O}_{17}$ : $\mathrm{Eu}^{2+}$ 沉积率最高, $\mathrm{CeMgAl}_{11} \mathrm{O}_{19}: \mathrm{Tb}^{3+}$ 和 $\mathrm{Y}_{2} \mathrm{O}_{3}: \mathrm{Eu}^{3+}$ 沉 积率较低，阳离子表面活性剂和非离子表面活性剂 PEG 能有效提高荧光粒子沉积率，过电位的不同反 映了三种荧光粒子润湿性的差异。

2) 三基色荧光粒子对涂层结构产生不同的影 响, $\mathrm{BaMgAl}_{11} \mathrm{O}_{17}: \mathrm{Eu}^{2+}$ 苂光粒子在涂层中出现少量 团聚和涂层结晶组织呈现节瘤状, $\mathrm{Y}_{2} \mathrm{O}_{3}: \mathrm{Eu}^{3+}$ 颗粒使 涂层镍的结晶取向由(200)晶面向(111)转变。

3) 三基色荧光粒子的添加，对涂层性能产生 不同影响, $\mathrm{Y}_{2} \mathrm{O}_{3}: \mathrm{Eu}^{3+}$ 颗粒使涂层晶粒细化, 涂层硬 度最高, $\mathrm{CeMgAl}_{11} \mathrm{O}_{19}$ : $\mathrm{Tb}^{3+}$ 粒子使涂层粗粘度最小, 硬度提高。

4) 通过对比复合涂层腐蚀前后的发光强度可 知，复合涂层起到了自敏指示的作用，其中蓝光 $\left(\mathrm{Ni}-\mathrm{BaMgAl}_{11} \mathrm{O}_{17}: \mathrm{Eu}^{2+}\right)$ 和绿光 $\left(\mathrm{Ni}-\mathrm{CeMgAl} \mathrm{H}_{11} \mathrm{O}_{19}\right.$ : $\mathrm{Tb}^{3+}$ )复合涂层在长期腐蚀环境中自敏指示性能良 好, 红光 $\left(\mathrm{Ni}-\mathrm{Y}_{2} \mathrm{O}_{3}: \mathrm{Eu}^{3+}\right)$ 复合涂层耐腐蚀性能较好, 但不具有长期指示作用。
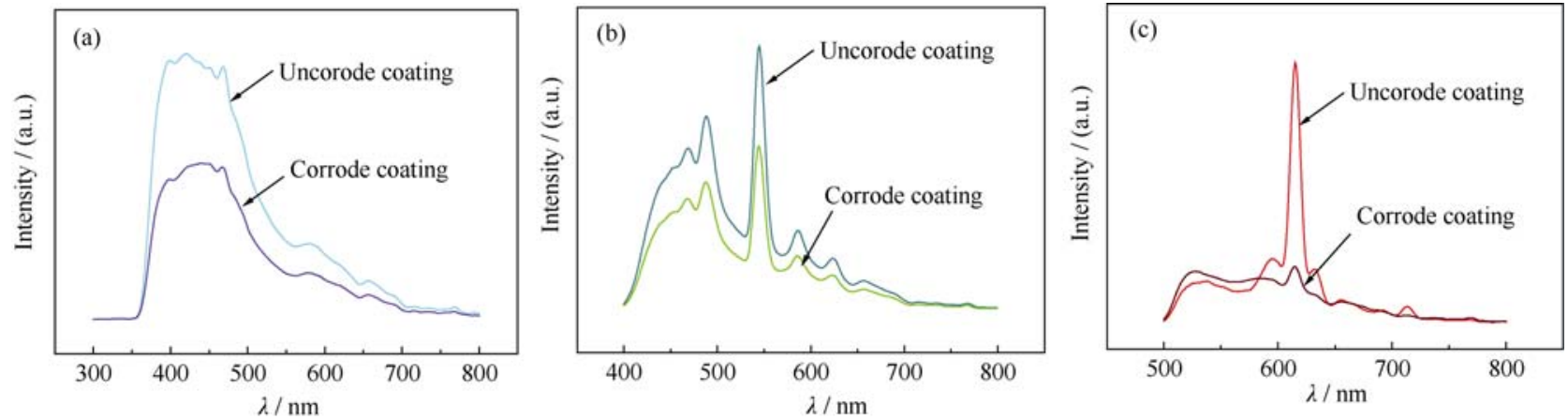

图 9 腐蚀前后三基色发光复合涂层的发光强度

Fig. 9 Emission spectra of composite coatings before and after corrode in the $3.5 \mathrm{wt} \% \mathrm{NaCl}$ solution (a) $\mathrm{Ni}-\mathrm{BaMgAl}_{11} \mathrm{O}_{17}: \mathrm{Eu}^{2+}$; (b) $\mathrm{Ni}-\mathrm{CeMgAl}_{11} \mathrm{O}_{19}: \mathrm{Tb}^{3+}$; (c) $\mathrm{Ni}_{-} \mathrm{Y}_{2} \mathrm{O}_{3}: \mathrm{Eu}^{3+}$ 


\section{参考文献:}

[1] GUTSEV D, ANTONOV M, HUSSAINOVA I, et al. Effect of $\mathrm{SiO}_{2}$ and PTFE additives on dry sliding of NiP electroless coating. Tribology International, 2013, 65(3): 295-302.

[2] BAKHIT B, AKBARI A, NASIRPOURI F, et al. Corrosion resistance of Ni-Co alloy and $\mathrm{Ni}-\mathrm{Co} / \mathrm{SiC}$ nanocompositecoatings electrodeposited by sediment codeposition technique. Applied Surface Science, 2014, 307(1): 351-359.

[3] ISLAM M, AZHAR M R, FREDJ N, et al. Electrochemical impedance spectroscopy and indentation studies of pure and composite electroless Ni-P coatings. Surface and Coatings Technology, 2013, 236(24): 262-268.

[4] HOUA K H, WANG H T, SHEU H H, et al. Preparation and wear resistance of electrodeposited $\mathrm{Ni}-\mathrm{W} /$ diamond composite coatings. Applied Surface Science, 2014, 308(1/2): 372-379.

[5] FELDSTEIN M D. Composite coatings with light-emitting properties. Metal Finish, 1999, 97(2): 87-90.

[6] ELDRIDGE J. Erosion-indicating thermal barrier coatings using luminescent sublayers. The American Ceramic Society, 2006, 89(10): $3252-3254$

[7] GANAPATHI M, ELISEEVA S V, BROOKS N R, et al. Electrodeposition of luminescent composite metal coatings containing rare earth phosphor particles. J. Mater. Chemistry, 2012, 22(12): $5514-5522$

[8] HE Y, WANG S C, WALSH F C, et al. The monitoring of coating health by in-situ luminescent layers. RSC Advance, 2015, 5(53): 42965-42970.

[9] LI QIANG, GAO LIAN, YAN DONG-SHENG, Luminescent charaeterization of nano- $\mathrm{Y}_{2} \mathrm{O}_{3}: \mathrm{Eu}^{3+}$. Journal of Inorganic Materials, 1997, 12(2): 237-241.
[10] LIN YING-LIANG, ZHOU GUANG-MEI, FENG DE-XIONG Preparation of the phoshor $\mathrm{BaMgAl}_{11} \mathrm{O}_{17}: \mathrm{Eu}^{2+}$ by the surface diffusion method. Journal of Inorganic Materials, 1999, 14(3): 425-430.

[11] LI JING-SHENG, SUN XUN-DONG, LI XIAO-DONG et al. (Y, Lu)A G: Ce phosphors synthesized by stearate melting method and theirfluorescence properties. Journal of Inorganic Materials, 2015, 30(2): 177-182.

[12] LI QIANG, GAO LIAN, YAN DONG-SHENG. Recent advance in nanoscale luminescent materials of rare earth compounds. Journal of Inorganic Materials, 2001, 16(1): 17-22.

[13] KILIC F, GULB H, ASLAN S, et al. Effect of CTAB concentration in the electrolyte on the tribological properties of nanoparticle $\mathrm{SiC}$ reinforced Ni metal matrix composite (MMC) coatings produced by electrodeposition. Colloids and Surfaces A: Physicochem. Eng. Aspects, 2013, 419(419): 53-60.

[14] QU N S, ZHU D, CHAN K C, et al. Fabrication of Ni-CeO $\mathrm{C}_{2}$ nanocomposite by electrodeposition. Scripta. Materialia, 2006, 54(7): $1421-1425$.

[15] PENG X, LI T., WU W, et al. Effect of $\mathrm{La}_{2} \mathrm{O}_{3}$ particles on the oxidation of electrodeposited nickel films. Oxidition Metals, 1999, 51(3/4): 291-315.

[16] PENG X, LI T, WU W, et al. Mater. Effect of $\mathrm{La}_{2} \mathrm{O}_{3}$ particles on microstructure and cracking-resistance of $\mathrm{NiO}$ scale on electrodeposited nickel films. Science Engineering, 2001, 298(1): 100-109.

[17] ZHOU Y B, SUN J F, WANG S C, et al. Oxidation of an electrodeposited $\mathrm{Ni}-\mathrm{Y}_{2} \mathrm{O}_{3}$ composite film. Corrosion Science, 2012, 63(10): 351-357.

[18] SHAKOOR R A, KAHRAMAN R, WAWARE U S, et al. Synthesis and properties of electrodeposited $\mathrm{Ni}-\mathrm{B}-\mathrm{CeO}_{2}$ composite coatings. Materials and Design, 2014, 59(59): 421-429.

[19] ZHOU XIAO WEI, SHENG YI PU. Corrosion behavior and EIS study of nanocrystalline $\mathrm{Ni}-\mathrm{CeO}_{2}$ coatings in an acid $\mathrm{NaCl}$ solution. Acta Metallurgica Sinica, 2013, 49(9): 1121-1130. 